\title{
EVOLUCIÓN DE LAS POLÍTICAS DE DESARROLlo SOCIAL EN MÉXico: ÉXITOS Y FRACASOS ${ }^{*}$
}

\author{
Gerardo Franco Parrillat ${ }^{* *}$ \\ Fabiola Canela Gambo* ${ }^{* * *}$
}

\section{Resumen}

La política social en México se ha venido fortaleciendo con la ampliación de cobertura y los cambios en el diseño del programa de transferencias condicionadas (PTC), así como con la creación de nuevos instrumentos de protección social, la mayoría de ellos con metas de cobertura universales o muy amplias. Al mismo tiempo, a pesar de los esfuerzos de las políticas de desarrollo territorial y de generación de oportunidades de ingreso, las condiciones de pobreza prevalecen, particularmente en los territorios con mayores rezagos. La escasa coordinación y vinculación entre los instrumentos de protección social, las políticas de inclusión productiva, las de desarrollo territorial y el PTC han limitado el éxito de estas estrategias a lo largo de los últimos quince años. Los más recientes cambios en el PTC parecen abrir una ventana de oportunidad para hacer efectiva la inclusión productiva de las familias beneficiarias de Prospera, considerando un enfoque territorial.

Palabras clave: pobreza, México, políticas públicas, enfoque territorial, inclusión productiva, protección social.

* Las opiniones y conclusiones expresadas en el artículo son de exclusiva responsabilidad de los autores y no necesariamente reflejan la posición del Centro Latinoamericano para el Desarrollo Rural (RIMISP).

** Maestro en Economía por El Colegio de México. Representante en México del Centro Latinoamericano para el Desarrollo Rural (RIMISP) (México).gfranco@rimisp.org

*** Maestra en Economía por la Universidad de las Américas-Puebla. Investigadora Adjunta del Centro Latinoamericano para el Desarrollo Rural (RIMISP) (México). fcanela@rimisp.org

Recibido: 9 de marzo de 2016 / Modificado: 8 de abril de 2016 / Aceptado: 3 de mayo de 2016

Para citar este artículo

Franco Parrillart, G. y Canela Gamboa, F. (2016). Evolución de las políticas de desarrollo social en México: éxitos y fracasos. opera, 18, pp. 159-181. DOI: http://dx.doi.org/10.18601/16578651.n18.09 


\section{EVOLUTION OF SOCIAL DEVELOPMENT POLICIES IN MEXICO: SUCCESSES AND FAILURES}

\section{Abstract}

The Social Policy in Mexico has been strengthened due to the expanded coverage and the modifications in the design of the Conditional Cash Transfer Programme (PTC for its name in Spanish), and to the creation of new social protection instruments with universal coverage as their main goal. Simultaneously, and despite the efforts of the territorial development policies and the generation of income opportunities, the poverty conditions prevail, specifically in those territories with higher backwardness. The lack of coordination and link-up among the instruments of social protection, the productive inclusion policies, those for territorial development and the PTC, have hindered the success of these strategies on the past fifteen years. Recent changes within the PTC seem to be an opportunity to reach the effective productive inclusion for beneficiary families of Prospera, considering a territorial approach.

Key words: Poverty, Mexico, public policies, territorial approach, productive inclusion, social protection.

\section{INTRODUCCIÓN: DE LA REVOLUCIÓN MEXICANA A PROGRESA}

La política de desarrollo social en México ha ido evolucionando desde las demandas sociales emanadas de la Revolución de 1910.
Diversos factores han provocado esta evolución, entre ellos las demandas sociales, las crisis, los modelos económicos y las nuevas teorías del desarrollo.

El primer avance significativo se presentó con la Ley Agraria de 1915 y el consecuente reparto de la tierra, lo cual generó las figuras de pequeños propietarios y titulares de derechos agrarios. Posteriormente, con la promulgación de la Constitución de 1917, se incorporan los derechos y las garantías individuales y el Estado asumió la responsabilidad de ser promotor del desarrollo nacional.

Con la llegada de Lázaro Cárdenas a la presidencia de la república, el reparto de tierras tomó un nuevo impulso y se elaboró el primer Plan Sexenal. No obstante, con la Segunda Guerra Mundial y el modelo de sustitución de importaciones implementado a partir de 1940, las prioridades se modificaron hacia el crecimiento económico y la ampliación de las coberturas de educación y salud, en particular en el sector urbano. Por otra parte, la política de desarrollo rural se vio mermada al focalizar sus acciones en zonas de agricultura comercial excluyendo del desarrollo a miles de campesinos - pequeños productores y de autoconsumo-.

Con la crisis de 1982, el país no pudo hacer frente a compromisos adquiridos, como la deuda externa, y el insuficiente presupuesto impidió enfrentar la demanda creciente de bienes y servicios públicos; con ello aumentaron los niveles de pobreza.

Los años noventa iniciaron con la implementación de las medidas y los postulados del Consenso de Washington. Un nuevo enfoque en la política social estaba emergiendo: la Ley 
Agraria de 1992 propiciaba la libre circulación de la tierra, se combinaron políticas sociales focalizadas con políticas asistencialistas y se crearon el Programa Nacional de Solidaridad (PRONASOL) y la Secretaría de Desarrollo Social (SEDESOL), en 1988 y 1992, respectivamente. La operación del PRONASOL fue inicialmente responsabilidad de la hoy extinta Secretaría de Programación y Presupuesto, y pasó a SEDESOL cuando esta institución nació.

Nuevamente, un escenario de crisis y cambios políticos obligó a la modificación de la política de desarrollo social: la crisis económica de 1995, que derivó en la devaluación del peso y el colapso de la economía del país, llevó al Gobierno de Ernesto Zedillo a reconocer en 1997 que todos los esfuerzos hechos habían sido insuficientes para combatir las causas estructurales de la pobreza, y a enfocar la política pública en dos vertientes: por un lado, la ejecución de acciones amplias dirigidas a la población en general en materia de seguridad y asistencia social, buscando con ello asegurar el disfrute de los derechos sociales, $y$, por el otro, acciones focalizadas dirigidas a la población en pobreza extrema. Una visión retrospectiva más amplia puede verse en Grupo de Diálogo Rural (2012).
Estimaciones posteriores ${ }^{1}$ del Consejo Nacional de Evaluación de la Política de Desarrollo Social (CONEVAL) dieron cuenta de que en 1996, el 53,5\% de la población rural se encontraba por debajo de la línea de pobreza alimentaria; $62,6 \%$ no contaba con ingresos suficientes para adquirir la canasta alimentaria y efectuar los gastos necesarios en salud y educación, aun dedicando el ingreso total de los hogares solo a estos fines, y $80,7 \%$ no disponía de ingresos suficientes para adquirir la canasta alimentaria, ni para realizar los gastos necesarios en salud, vestido, vivienda, transporte y educación.

Dada esta situación, y con el propósito de aumentar las capacidades de la población, en 1997 se puso en marcha el Programa de Educación, Salud y Alimentación (Progresa), donde se conjugaron subsidios alimentarios y acciones de salud y educación para asegurar la entrega de un paquete básico, poniendo énfasis en las mujeres y los nińos en condiciones de pobreza extrema en el ámbito rural. Con una cobertura inicial de 300 mil familias ${ }^{2}$, el programa se expandió hasta alcanzar 2,4 millones de las familias (Levy y Rodríguez, 2005).

1 En 2002, el Gobierno organizó el Comité Técnico para la Medición de la Pobreza (en adelante Comité), que aglutinó a especialistas académicos y gubernamentales para establecer una metodología consensuada para la medición oficial de la pobreza en México. Esta medición permaneció vigente hasta la creación, en 2004, de la Ley General de Desarrollo Social, que modificó los criterios para la medición de la pobreza con características multidimensionales. Actualmente, las mediciones de pobreza por ingresos, según los criterios del Comité, siguen reportándose por su importancia como serie histórica.

2 Según estimaciones del coneval, en 1996 existían 22,7 millones de personas por debajo de la línea de capacidades, es decir, que no contaban con ingresos suficientes para adquirir el valor de la canasta alimentaria y efectuar los gastos necesarios en salud y educación. 
FIGURA 1. EVOLUCIÓN DE LA POBREZA RURAL ANTES Y DESPUÉS DE LA CRISIS DE 1995

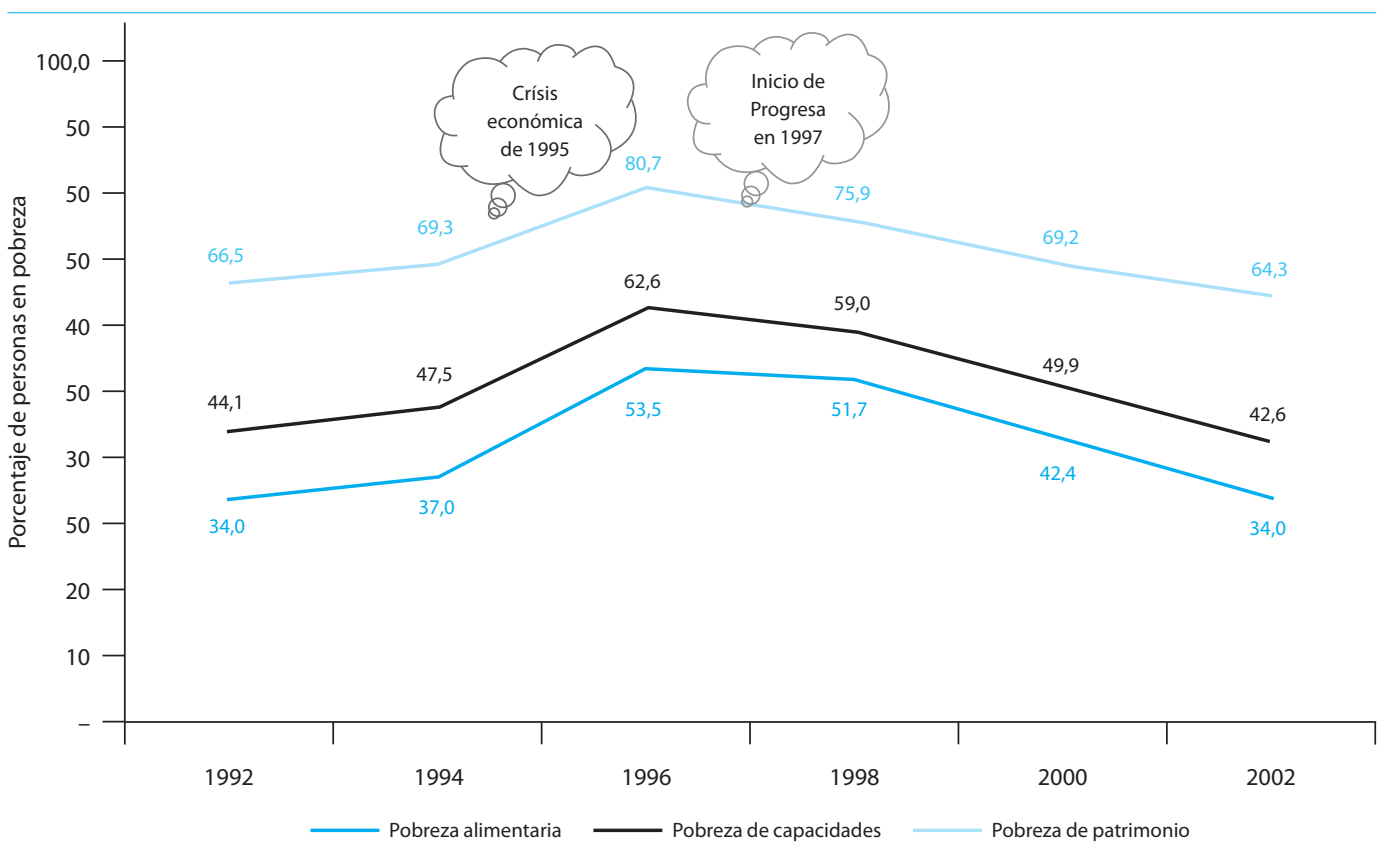

Este programa de transferencias condicionadas (PTC) forma parte de la entonces denominada "nueva generación" de programas de lucha contra la pobreza enfocados a promover la acumulación de capital humano en los hijos de las familias en situación de pobreza, para romper los ciclos intergeneracionales de pobreza, considerando que una persona más sana, mejor alimentada y con mayor educación podría acceder a mejores opciones dentro del mercado de trabajo. Además, desde su diseño, se incluyó una rigurosa evaluación de impacto que mostró efectos positivos en el aumento en la matrícula de educación primaria y secundaria, la disminución del trabajo infantil, el control nutricional y la vacunación, el crecimiento infantil, un mayor consumo alimentario promedio del hogar (principalmente de frutas, verduras y productos animales), así como un aumento en la ingesta calórica, según Rawlings y Rubio (2003). Se piensa que estos resultados, y la comprensión de que los efectos en capital humano requerían la implementación de mediano plazo del programa, fueron fundamentales para las etapas posteriores de Progresa, en un escenario de alternancia política que podría haber concluido con el programa.

\section{ESTRATEGIA CONTIGO ES POSIBLE:} UNA POLÍTICA DE LUCHA CONTRA

\section{LA POBREZA}

Con la alternancia de los gobiernos se modificaron también las políticas públicas. El presidente Vicente Fox (2000-2006) im- 
pulsó la denominada Estrategia Contigo es Posible, la cual fue una política de desarrollo social que buscaba romper el círculo vicioso del empobrecimiento. El enfoque se planteaba como un cambio conceptual de la pobreza entendida únicamente como consecuencia de los bajos ingresos de la población, a otro en el que se ponía énfasis en la ampliación de capacidades, la promoción de la protección social, la generación de oportunidades de ingreso y la formación de patrimonio.

Con esta política, por primera vez se integró en una sola estrategia nacional la acción de quince dependencias de la administración pública federal. Inicialmente consideró la integración de 223 programas federales existentes, los cuales se agruparon en torno a las cuatro vertientes:

- Ampliación de capacidades, que incluía educación, salud y nutrición, y capacitación: 123 programas.

- Protección social, que incluía previsión social y protección contra riesgos individuales y colectivos: nueve programas.

- Generación de oportunidades de ingreso, que incluía desarrollo local y acceso al crédito, así como generación de empleo: 38 programas.

- Formación de patrimonio, que incluía ahorro, vivienda y derechos de propiedad: 53 programas.

Esta estrategia buscaba atender las causas estructurales de la pobreza en todo el ciclo de vida de los individuos y las familias, con acciones encaminadas a la atención prenatal, la infancia, la adolescencia, la edad adulta y los adultos mayores. Durante el sexenio, fue necesario hacer cambios en la estructura programática, eliminar programas que no estaban alineados a la nueva estrategia y modificar o crear otros programas. A continuación, se presentan algunos de los programas emblemáticos de la Estrategia Contigo, con un enfoque de desarrollo social.

Oportunidades. A partir de 2002 Progresa se transformó en el Programa de Desarrollo Humano Oportunidades (en adelante Oportunidades) y se erigió como un programa emblemático de la Estrategia Contigo para la ampliación de capacidades. En este contexto, se mantuvieron los esquemas operativos empleados y las principales características de Progresa; se amplió el número de beneficiarios incluyendo familias de localidades urbanas, y se integraron nuevas acciones, con el fin de ampliar el bienestar de las familias en condición de pobreza. A mediados de 2002, el programa atendía a poco más de cuatro millones de hogares. Ese mismo año se extendió a los 32 estados del país, y el apoyo educativo creció: las becas a educación básica se ampliaron hasta educación media superior.

Seguro Popular. La protección social impulsada desde la Estrategia Contigo es Posible consistió en un conjunto separado de beneficios para los trabajadores del sector informal, siendo el Seguro Popular el que mayor cobertura logró. Con la reforma de 2002 a la Ley General de Salud se reconoció la protección social en salud, y en mayo de 2003 se creó la Comisión Nacional de Protección Social en Salud de la Secretaría de Salud (ssa). Esta Comisión es la responsable de financiar la provisión de los servicios 
FIGURA 2. ESTRATEGIA CONTIGO: CICLO DE VIDA Y ESTRATEGIAS ARTICULADAS

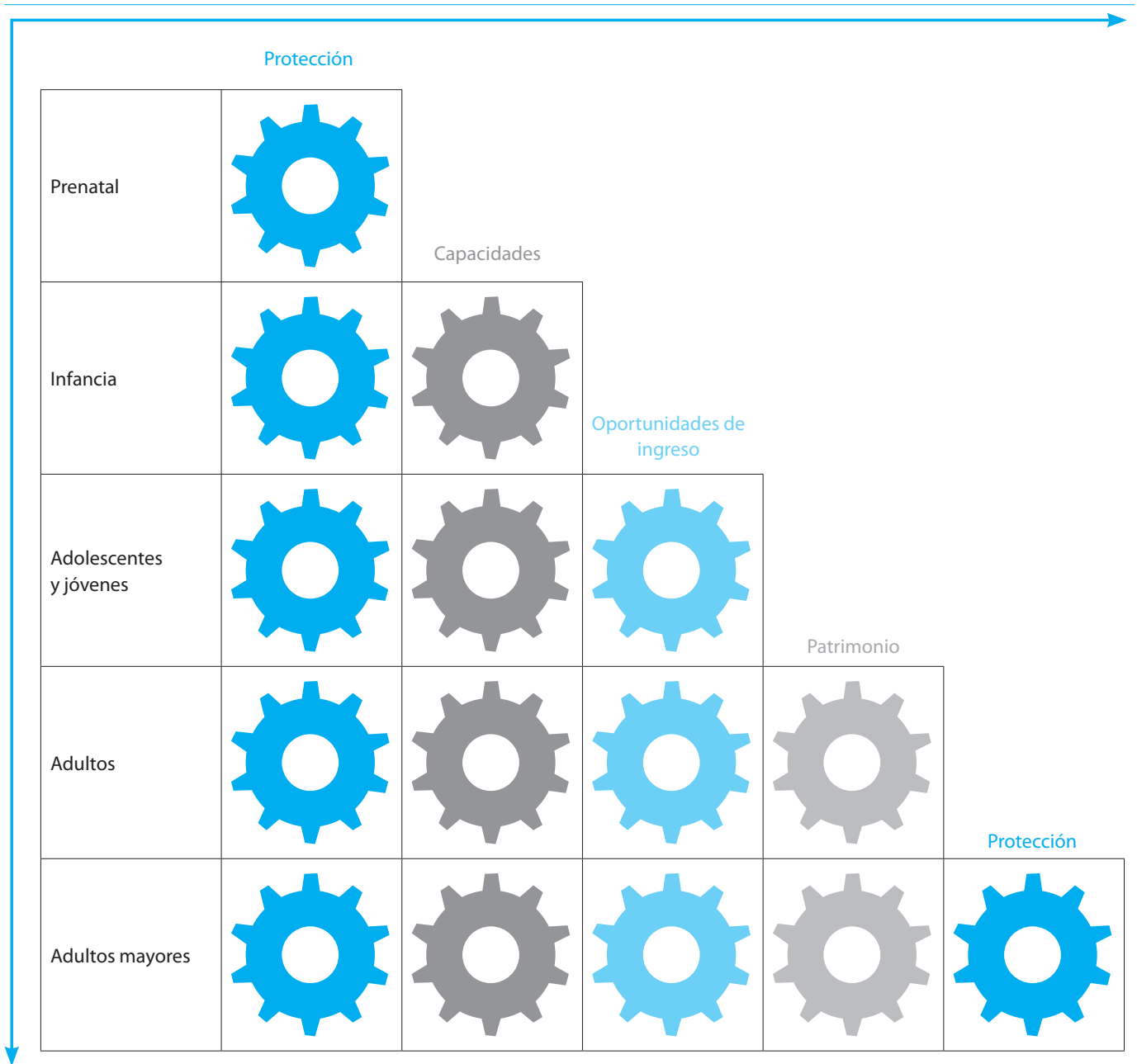

de salud a la población beneficiaria del sistema de protección social en salud. El Seguro Popular forma parte del sistema y tiene como población objetivo a las personas no afiliadas a servicios de seguridad social como el Instituto Mexicano del Seguro Social (IMSs) o el Instituto de Seguridad y Servicios Sociales de los Trabajadores del Estado (ISSSTE), para evitar que realicen gastos catastróficos por atender problemas de salud.

Estrategia Microrregiones. El 14 de febrero de 2001 se puso en marcha la Estrategia de Microrregiones como un mecanismo de atención a las disparidades regionales, para vincular la producción, la distribución y el consumo, y promover un desarrollo regional 
productivo sostenido. En la estrategia participaban 68 programas federales de 12 dependencias para atender a los mil 338 municipios de alta y muy alta marginación, con prioridad en dos mil 966 localidades denominadas Centros Estratégicos Comunitarios (CEC) (Mujica, 2007) que, por su potencial en infraestructura y ubicación, eran considerados con capacidad de ofrecer servicios básicos a localidades pequeñas y dispersas. En estos CEC, se buscaba asegurar los servicios de electricidad, salud, telefonía, caminos, saneamiento, educación, agua potable, abasto de productos básicos y piso firme, así como Centros Comunitarios de Aprendizaje y, principalmente, la existencia de circuitos de actividad productiva ${ }^{3}$.

En cuanto a la formación de patrimonio, la Estrategia Contigo no hizo grandes cambios. Los dos principales programas de vivienda se ejecutaron desde el fideicomiso Fondo Nacional de Habitaciones Populares (FONHAPO), creado en 1981 como una alternativa de financiamiento para atender las necesidades de vivienda de las familias de menores recursos económicos. El Programa de Ahorro, Subsidio y Crédito para la Vivienda Progresiva presentó algunas adecuaciones menores respecto del diseño inicial de 1998. Por otra parte, en 2004 se creó un nuevo Programa de Vivienda Rural, en el marco de los compromisos del Acuerdo Nacional para el Campo (ANC), firmado en abril de 2003 por el Gobierno Federal y organizaciones campesinas integradas en el movimiento "El campo no aguanta más". Ese movimiento argumentaba que la mayor desgravación de productos agrícolas pactada en el Tratado de Libre Comercio de América del Norte (TLCAN) a partir de 2003 traería graves repercusiones al sector agrícola, y desde finales de 2002 realizó manifestaciones y ejerció diversas formas de presión hasta lograr una negociación con el Gobierno Federal que derivó en el ANC.

Sin embargo, a pesar de que Contigo era una estrategia integral de desarrollo social, pocas posibilidades tenía para lograr una adecuada vinculación, incluso entre los programas pertenecientes al mismo sector, porque las reglas de operación de la Secretaría de Desarrollo Social no establecían criterios específicos para atender de forma integral a las familias de Oportunidades con los otros programas de la estrategia.

Un ejemplo particular se presentó en el Programa de Oportunidades Productivas, creado en 2002 y que en su nombre parecía establecer una vinculación con los beneficiarios de Oportunidades. En realidad, este programa no establecía lazo alguno con el PTC, pues sus reglas de operación señalaban como población objetivo "Hombres, mujeres, unidades familiares y grupos sociales en pobreza extrema que deseen obtener apoyos a la palabra, diversificar su actividad económica, obtener un crédito social para consolidar proyectos en operación, desarrollar proyectos productivos y obtener capacitación y asistencia técnica en procesos productivos y organizacionales". Incluso un

3 Estas acciones constituían las denominadas "Banderas blancas", que eran los elementos mínimos necesarios para detonar el desarrollo territorial. 
ańo más tarde, su nombre se modificó a Opciones Productivas, dejando de lado cualquier relación con el PTC.

Otro ejemplo es la escasa o nula vinculación entre el PTC y el Seguro Popular. Si bien es cierto que a finales de 2005 un total de tres millones 555 mil 977 familias estaban afiliadas al Seguro Popular y que alrededor de $39,8 \%$ pertenecía al programa Oportunidades (Comisión Nacional de Protección Social en Salud, 2005), esta cobertura era más bien casual y no planificada con la intención de contar con políticas integrales, pues las decisiones de la cobertura recaían en las entidades federativas, que son las responsables a nivel local de la afiliación de beneficiarios, administración de los recursos, supervisión de los prestadores de servicios y pago de los mismos. Así, en los primeros años, las entidades no establecieron obligaciones o prioridades respecto de los beneficiarios del PTC. En 2014, el Seguro Popular atendió a 57 millones 300 mil beneficiarios.

\section{ESTRATEGIA VIVIR MEJOR: UNA POLÍTICA DE DESARROLLO HUMANO SUSTENTABLE}

Durante la administración del residente Calderón (2006-2012) se impulsó la estrategia Vivir Mejor, cuyo objetivo era concentrar las acciones hacia el desarrollo humano sustentable. En la estrategia, este concepto era entendido como la ampliación de las capacidades que permiten a las personas acceder a una vida digna sin comprometer el patrimonio de las generaciones futuras. Vivir Mejor se basaba en la atención coordinada de las acciones del Gobierno, evitando la dispersión y la subu- tilización de los recursos públicos. Señalaba también las necesidades de complementariedad entre los tres órdenes de Gobierno (federal, estatal y municipal) para alcanzar los objetivos planteados.

Esta estrategia reconocía que no solo hay que corregir la brecha entre pobres y ricos, sino también entre la armonía de la naturaleza y el hombre. Valoraba además la organización comunitaria como una instancia fundamental para el reforzamiento de la cohesión social. La estrategia consideraba tres líneas de acción para el desarrollo humano sustentable:

- Impulsar el desarrollo de las capacidades de los mexicanos, en especial de las niñas y los nińos, garantizándoles acceso a educación, salud y vivienda digna.

- Proporcionar una red de protección social que evitara el empobrecimiento extremo en las familias más vulnerables, ante la presencia de enfermedades o la pérdida del empleo.

- Facilitar el acceso al empleo formal a todas las mexicanas y los mexicanos, fortaleciendo la coordinación entre la política social y la política económica, mediante el establecimiento de puentes comunicadores hacia un desarrollo económico sostenido y sustentable.

Como instrumentos de estas líneas de acción, se conservaron varios de los programas emblemáticos creados en el sexenio anterior, como el Seguro Popular y el programa Oportunidades. En este último se realizaron algunas modificaciones para fortalecer la red de protección social de las familias del pro- 
grama, en respuesta al alza sostenida en los precios internacionales de los alimentos en 2007-2008. Se creó entonces el componente de apoyo alimentario Vivir Mejor, consistente en un auxilio económico transitorio para compensar la disminución del poder adquisitivo de las familias de Oportunidades, las cuales realizaban cerca del $50 \%$ de su gasto total en alimentos. No obstante que en su diseño se establecía como un apoyo transitorio, posteriormente el mismo se incorporó a la transferencia condicionada habitual para alimentación.

Se intentó también otro esquema de protección social a partir de 2006, al incluir en Oportunidades el Mecanismo de Ahorro para el Retiro Oportunidades (MAROP), un subsidio destinado al ahorro para el retiro entre la población que no disponía de empleo formal, y cuya finalidad era complementar el ahorro individual para que los adultos mayores de las familias beneficiarias de Oportunidades pudieran contar con recursos en cuanto llegaran a los 70 años de edad para adquirir una renta vitalicia que contribuyera a mejorar su nivel de vida. El MAROp fue abrogado en 2008 dado que, con miras a obtener un ingreso futuro, ocurría una reducción del ingreso presente disponible para los beneficiarios del mecanismo, lo cual tenía un efecto negativo en el bienestar familiar. Ante estas limitantes, se optó por avanzar hacia un sistema de pensión universal no contributiva.

Programa 70 y Más. Uno de los cambios más importantes para consolidar una red de protección social se introdujo con la ampliación de la pensión no contributiva para adultos mayores. Entre 2001 y 2006, las confrontaciones entre el entonces jefe de Gobierno del Distrito Federal y el presidente de la república eran recurrentes y ello repercutió en decisiones de política pública. En 2001, el Gobierno del Distrito Federal inició el programa de Apoyo Alimentario a Adultos Mayores, y en 2003 se aprobó la Ley que Establece el Derecho a la Pensión Alimentaria para Adultos Mayores Residentes en el Distrito Federal. En respuesta, el Gobierno Federal implementó ese mismo año el programa de Atención a Adultos Mayores enfocado a las personas con edades a partir de 60 años, de localidades rurales con menos de dos mil 500 habitantes, de alta y muy alta marginación.

En 2007 se aumentó a 70 años y más la edad de elegibilidad de este último programa y se eliminó la condicionante de vivir en localidades de alta y muy alta marginación. En 2008 se amplió la cobertura a localidades de hasta 20 mil habitantes, en 2009 se estableció el límite en 30 mil habitantes, y a partir de 2012 involucró a las localidades de más de 30 mil habitantes, lo cual orientó una aplicación universal de sus beneficios. Este programa otorga transferencias monetarias para mejorar el ingreso de los adultos mayores con 70 años o más que carecen de una pensión contributiva y no están afiliados a una institución de seguridad social.

En 2010, la evaluación de impacto del programa señaló que la transferencia bimestral de mil pesos (que representa $50 \%$ del ingreso del hogar de los beneficiarios) impulsó la reducción en $18 \%$ de la proporción de adultos mayores que trabajaban por pago. Esto se tradujo en una mayor dedicación a actividades familiares, sin que esto mermara 
los ahorros del hogar, y en una reducción del tiempo destinado a movilizarse hacia el trabajo. A pesar de que el programa disminuye el ingreso laboral, la transferencia monetaria permite que se incremente el consumo en $24 \%$. Asimismo, los evaluadores recomendaron que el programa se extendiera a otras comunidades rurales. Sin embargo, la extrapolación de estos resultados a comunidades más grandes no era aconsejable debido a las diferencias poblacionales.

Actualmente, el programa se focaliza en adultos mayores de 65 años y se denomina Pensión para Adultos Mayores. Al quinto bimestre de 2015, el programa atendió a cinco millones 724 mil 854 adultos mayores ${ }^{4}$.

Programa de Guarderías y Estancias Infantiles. Este programa fue emblemático para la red de protección social de la estrategia Vivir Mejor. Se creó con el objetivo de facilitar la inserción de las madres y los padres trabajadores en el mercado laboral y mejorar sus ingresos, garantizando que sus hijos menores de seis años fueran atendidos en condiciones dignas y seguras, y superar la disyuntiva entre la búsqueda de empleo, el cuidado de los hijos y los gastos en guarderías o cuidadores particulares durante la búsqueda de empleo o las jornadas laborales. Además, sin importar si la incorporación al empleo se da en el mercado laboral formal o informal, existe una insuficiente oferta para cubrir la demanda por este servicio. Por ello, el programa otor- ga subsidios a los servicios particulares de cuidado y atención infantil, así como apoyos para incrementar la oferta de estos servicios.

El programa fue objeto de una evaluación de impacto que dio como resultado que las titulares de las estancias registraran un aumento de $21 \%$ en empleo, un incremento de poco más de siete horas de trabajo a la semana y un aumento de $16 \%$ en la permanencia laboral a corto plazo.

Estrategia 100x 100. Para facilitar los puentes entre la política social y la política económica, particularmente en los territorios más desfavorecidos, se creó en 2007 la Estrategia $100 \times 100$, que buscaba transformar las condiciones de rezago de los municipios con menor Índice de Desarrollo Humano (IDH) para impulsar su desarrollo integral, social y económico, por medio del mejoramiento de las condiciones de vida de la población y del incremento de las posibilidades para una mayor productividad y empleo. Esta estrategia ratificó la importancia de la coordinación entre los tres niveles de gobierno, la sociedad civil y los organismos públicos y privados en los 125 municipios participantes. Su operación estuvo a cargo de la Comisión Intersecretarial de Desarrollo Social, con la intención de evitar los problemas de coordinación ocurridos en la Estrategia Microrregiones de la anterior administración.

Durante 2007-2011 se realizaron inversiones convergentes por 39 mil 544 millones

4 Portal de transparencia presupuestaria de la Secretaría de Hacienda y Crédito Público. Documento "Cobertura de las poblaciones potencial, objetivo y atendida de los programas sujetos a Reglas de Operación", datos al quinto bimestre de 2015. 
de pesos en los 125 municipios de la estrategia. Sin embargo, $87 \%$ de los recursos provinieron del Gobierno federal y solo el $13 \%$ de los estados y municipios (CONEVAL, 2013b).

La evaluación realizada por el CONEVAL concluyó que el conjunto de las inversiones obtuvo efectos sobre la población sin derecho a servicios de salud y porcentaje de viviendas con piso de tierra. Sin embargo, la evaluación plantea que las acciones de los programas federales coordinadas por la estrategia no se concentraron solo en los 125 municipios destinatarios, es decir, no se cumple la oportuna priorización en la atención ofrecida.

Los resultados obtenidos en esta evaluación y en la correspondiente a la Estrategia
Microrregiones sugieren que para lograr un mayor impacto en las políticas con enfoque regional se requiere que el diseño asegure la priorización, la coordinación institucional y el acoplamiento entre los distintos niveles del Gobierno.

Otro elemento de valoración importante de los avances en la política social en la administración del presidente Calderón se presentó con la medición multidimensional de la pobreza. Esta metodología, establecida por el Coneval con base en la Ley General de Desarrollo Social, y cuya primera medición se realizó para 2008, dio cuenta de los avances en la cobertura de los derechos sociales establecidos en la ley: acceso a la alimentación, salud,

FIGURA 3. EVOLUCIÓN DE LAS CARENCIAS SOCIALES 2008-2012

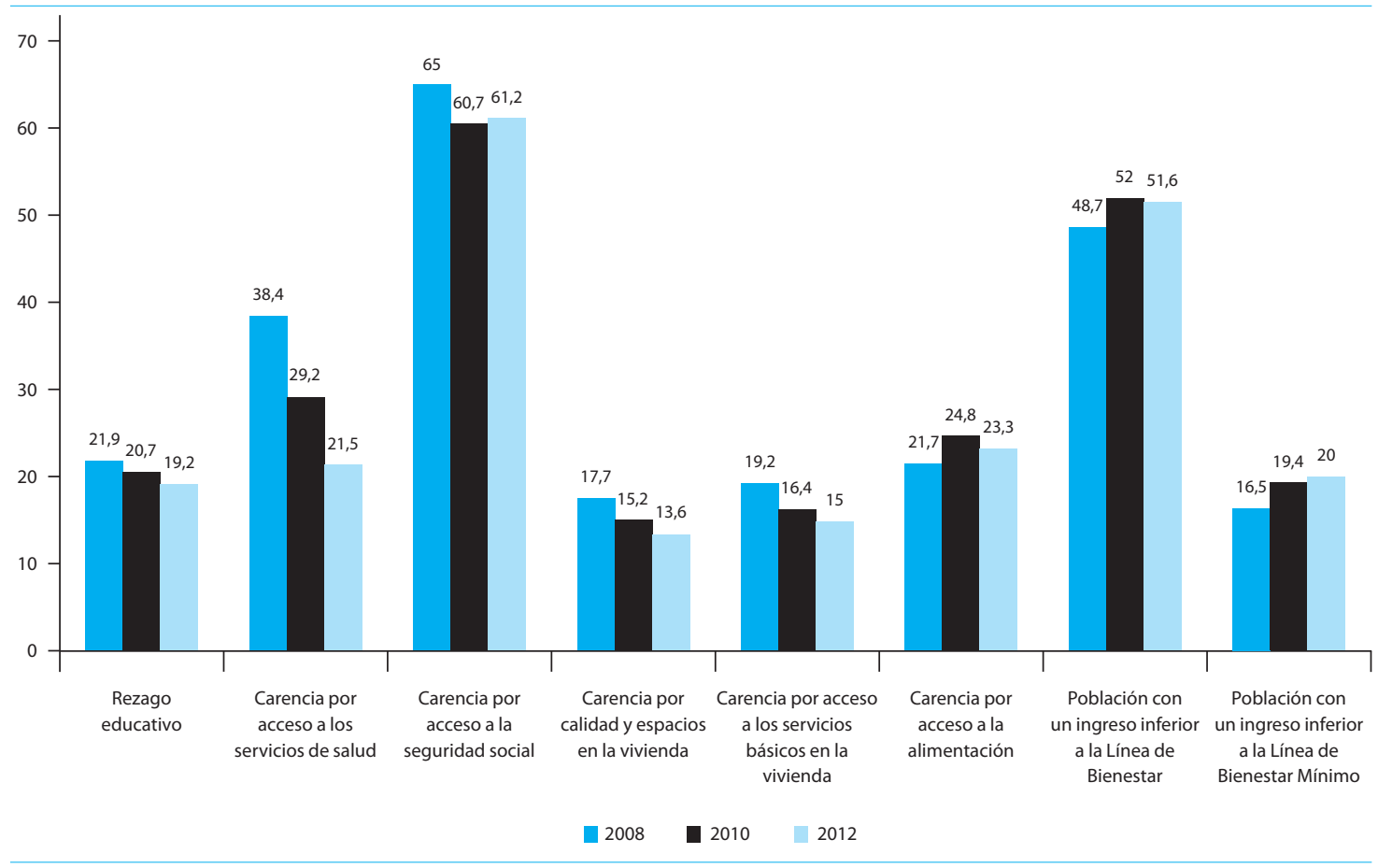

Fuente: elaboración propia con datos del CONEVAL. 
educación, calidad y espacios de la vivienda, servicios básicos en la vivienda, seguridad social e ingresos.

En general, los resultados fueron alentadores para cinco de los seis indicadores de carencia que disminuyeron entre 2008 y 2012, a excepción del acceso a la alimentación. A pesar de la expansión del Seguro Popular, la Pensión para Adultos Mayores y la creación de nuevos elementos de seguridad social, este indicador se encuentra ampliamente ligado a los beneficios que otorga el empleo en el mercado laboral formal, por lo que los instrumentos de protección social desarrollados no han sido suficientes.

Además de los elevados niveles del indicador de seguridad social y el incremento en la carencia de seguridad alimentaria, el aumento en los niveles de pobreza se debe al crecimiento de la población con ingresos inferiores a la línea de bienestar. La implementación de puentes entre la política social y la política económica, que representaba una línea de acción de la Estrategia Vivir Mejor, no fue suficiente para evitar el incremento de la población en condiciones de pobreza.

\section{CRUZADA NACIONAL CONTRA EL HAMBRE: UNA ESTRATEGIA DE INCLUSIÓN Y BIENESTAR SOCIAL}

El presidente Enrique Peña Nieto, que se posesionó el 1 de diciembre de 2012, se comprometió a encabezar una transformación de México basada en trece decisiones presidenciales, una de ellas fue la encomienda a la Secretaría de Desarrollo Social de coordinar la Cruzada Nacional Contra el Hambre (CNCH).
A principios de 2013 se publicó en el Diario Oficial de la Federación el decreto por el que se crea el Sistema Nacional para la Cruzada contra el Hambre (sinhambre), el cual señala:

La Cruzada contra el Hambre es una estrategia de inclusión y bienestar social, que se implementará a partir de un proceso participativo de amplio alcance cuyo propósito es conjuntar esfuerzos y recursos de la Federación, las entidades federativas y los municipios, así como de los sectores público, social y privado y de organismos e instituciones internacionales, para el cumplimiento de los objetivos a que se refiere el artículo Segundo del presente Decreto.

La población objetivo de la Cruzada contra el Hambre, de acuerdo con las estimaciones del coneval y con la Encuesta Nacional de Ingresos y Gastos de los Hogares 2010 (ENIGH, 2010), ascendía a 7,4 millones de personas que viven en condiciones de pobreza multidimensional extrema y simultáneamente presentan carencia por acceso a la alimentación.

A diferencia de las estrategias anteriores, el marco conceptual que genera la Cruzada está relacionado con los derechos sociales marcados en la Ley General de Desarrollo Social. Esto implica la priorización del grupo meta representado por la población en pobreza extrema, es decir, mexicanos y mexicanas con tres o más derechos sociales no cubiertos, uno de los cuales es el derecho a la alimentación, e ingresos inferiores a la línea de bienestar.

Sin embargo, a pesar de lo anterior, los objetivos de la Cruzada planteados en el decreto por el que se establece sinHAmbre consideran únicamente el derecho a la ali- 
mentación y la mejora en los ingresos de e sta población:

- Cero hambre a partir de una alimentación y nutrición adecuadas de los mexicanos en extrema pobreza y con carencia alimentaria severa.

- Eliminar la desnutrición infantil aguda y mejorar los indicadores de crecimiento de niños y niñas en la primera infancia.

- Aumentar la producción y el ingreso de los pequeños productores agrícolas.

- Minimizar las pérdidas poscosecha y de alimentos durante el almacenamiento y transporte, así como en los comercios.

- Promover la participación comunitaria.

Para lograr sus objetivos, la Cruzada se implementaría en dos etapas; la primera, enfocada en 400 municipios seleccionados con base en la incidencia de personas en situación de pobreza extrema y con carencia por acceso a la alimentación;la segunda plantea su extensión a todo el país 5 .

Dado que su implementación implica un proceso participativo entre el Gobierno Federal, estados, municipios y sectores público, social y privado, así como de organismos e instituciones internacionales, se creó la Comisión Intersecretarial, integrada por 19 secretarías y 90 programas; la Comisión Nacional para el Desarrollo de los Pueblos Indígenas (CDI) el Instituto Nacional de las Mujeres (INMUjeres) y el Sistema Nacional para el Desarrollo Integral de la Familia (DIF), con el fin de coordinar, articular y complementar las acciones, los programas y recursos necesarios para el cumplimiento de los objetivos de la Cruzada. Adicionalmente, se creó el Consejo Nacional de la Cruzada Contra el Hambre, instancia en la que convergen los sectores público, privado y social.

En 2014, se logró identificar a 5,5 millones de personas en pobreza extrema con carencias de alimentación en el territorio nacional, de las cuales 4,2 millones están siendo atendidas al menos por un programa social de la Cruzada (Secretaría de Desarrollo Social, 2015b). El conEval, en su Informe de resultados intermedios, específicamente en el estudio exploratorio del impacto, observa una reducción significativa de dos puntos porcentuales en la pobreza extrema. Sin embargo, no registra efecto en el indicador de carencia por acceso a la alimentación. Por otro lado, las particularidades de la Cruzada como estrategia de coordinación y participación y las fórmulas para identificar a la población beneficiaria plantean retos para la implementación de una metodología para medir su impacto.

Como se mencionó, la Cruzada es una articulación de coordinación y participación de diversas Secretarías de Estado que suman esfuerzos por medio de sus programas para el logro de los objetivos planteados. Específicamente, hay tres programas emblemáticos de esta estrategia -dos de los cuales surgieron en esta administración-que están focalizados a contribuir a la política social orientada a reducir las carencias sociales. En especial, las

5 Para 2014, la Cruzada se amplió a 1.012 municipios (Secretaría de Desarrollo Social, 2015b). 
acciones de la Cruzada han focalizado apoyos a hogares en pobreza extrema (con una población objetivo de siete millones de personas) y han logrado reducir las carencias sociales de esa población. Sin embargo, se deben encontrar estrategias para lograr la reducción de la pobreza más amplia que involucra a 55 millones de personas (CONEval, 2015).

Programa de Comedores Comunitarios. La Secretaría de Desarrollo Social creó este programa con el propósito de incrementar el acceso a alimentos en los territorios urbanos y rurales ubicados en las Zonas de Atención Prioritaria. Con este programa se pretende mejorar las condiciones nutricionales de la población de niños y niñas de cero a 11 años de edad, mujeres en gestación y en lactancia, así como personas con alguna discapacidad y adultos mayores de 65 años.

El programa, además, promueve el desarrollo de organización comunitaria y el diseño de procedimientos que permitan que la población se involucre y se responsabilice de la operación de los Comedores para atender a las personas que asisten a los mismos. Asimismo, los Comedores están planteados como lugares incluyentes, donde se fomenta la relación familiar y social, la sana convivencia, la comunicación, el encuentro y la participación como atributos básicos de la cohesión social.

Programa Seguro de Vida para Jefas de Familia. La presente administración identifica como grupo vulnerable de protección social a los hijos de jefas de familia que presentan carencias por acceso a la seguridad social, por lo que impulsa el programa Seguro de Vida para Jefas de Familia. Este programa tiene como objetivo contribuir a la ampliación del sistema de seguridad social mediante la incorporación de las jefas de familia de entre 12 y 68 ańos de edad que presentan carencias por acceso a la seguridad social. A partir del 2015, su condicionamiento cambió a aquellas que estén en situación de vulnerabilidad. Ante el fallecimiento de la jefa de hogar, el programa otorga mensualmente apoyos económicos directos a las hijas y los hijos, incentivando su ingreso o permanencia en el sistema escolar hasta los 23 años de edad. Los montos de los apoyos varían según el nivel educativo al cual asista el nińo(a) o el o la joven.

Prospera. Antes denominado Programa de Desarrollo Humano Oportunidades, el Programa de Inclusión Social (Prospera), además de apoyar en alimentación, salud y educación, articula esfuerzos para lograr la inclusión financiera, laboral y productiva de las personas por medio de su propio trabajo. Prospera coordina la oferta institucional de programas y acciones de política social, incluyendo aquellas relacionadas con el fomento productivo, la generación de ingresos, el bienestar económico, la inclusión financiera y laboral, la educación, la alimentación y la salud, dirigida a la población que se encuentre en situación de pobreza.

Este nuevo programa no solo asegura la asistencia social; también promueve el bienestar de los hogares facilitando el acceso al mercado laboral y productivo, lo que implica vinculación y coordinación entre la política social y la política económica. Antes de esta iniciativa, la población en condición de pobreza no tenía acceso a programas productivos, porque se encontraba en localidades relativamente aisladas y porque se pensaba que las 
personas en situación de pobreza carecían de potencial de respuesta a estímulos de orden productivo y que, por tanto, la superación de su condición debía lograrse por la vía de políticas sociales. De ahí la disociación de las políticas de desarrollo productivo y de desarrollo social.

En ese contexto surge el Programa Territorios Productivos (PTP), que vincula la política de desarrollo económico y la política social mediante desarrollo territorial. Este programa piloto está orientado a hogares beneficiarios de Prospera que se ubican en municipios donde está presente la Cruzada.

El PTP tiene como objetivo contribuir a reducir la población rural en condición de pobreza extrema, por medio del aumento de su productividad, producción e ingresos autónomos, en congruencia con uno de los objetivos previstos de la política de desarrollo social: "Mejorar el ingreso de las personas en situación de vulnerabilidad mediante el apoyo y desarrollo de proyectos productivos". El PTP busca que los hogares rurales en condición de pobreza accedan efectivamente a las políticas y programas públicos disponibles, y se basa en la evidencia de que -en México y en otros países (incluso con menor nivel de desarrollo)- con adecuados diseños de política e instrumentos apropiados, los habitantes rurales en condición de pobreza pueden incrementar su productividad o su vinculación a trabajos de mejor calidad, y de esta forma elevar sus ingresos.

Este programa beneficiará a 10 mil personas en cinco estados de México, y se enfocará en articular, con una visión territorial, los apoyos de 15 programas productivos dispo- nibles en diversas dependencias del Gobierno Federal.

\section{DESARROLLO DE CAPACIDADES, PROTECCIÓN SOCIAL, INCLUSIÓN PRODUCTIVA Y DESARROLLO TERRITORIAL}

Tassara, Ibarra y Vargas (2015) sugieren revisar la Estrategia de Inclusión Activa (EIA) de la Unión Europea por sus similitudes con la evolución de los PTC. Dicha EIA define tres pilares de la inclusión social: 1) la existencia de un apoyo al ingreso coherente con el costo de la vida; 2) la presencia de un mercado laboral que ofrezca oportunidad de empleo y generación de ingresos; y 3 ) el acceso a servicios sociales de buena calidad.

Las transformaciones que ha tenido el PTC en México, hasta generar el actual Prospera, han apuntado a conformar estos tres pilares. Por una parte, la cuantía de las transferencias monetarias se ha venido reajustando, tanto en casos de contingencias - por ejemplo, frente a la crisis mundial de los precios de los alimentos- como en forma permanente, de acuerdo con la disponibilidad presupuestaria y con base en la variación acumulada de los índices asociados a las líneas de bienestar mínimo (rural y urbano), publicadas por el CONEVAL.

Por otra parte, los cambios de Oportunidades a Prospera buscan atender las recomendaciones del Coneval para:

\footnotetext{
...(a) destinar recursos a programas existentes con mayor incidencia sobre poblaciones pobres (con potencial productivo); (b) revisar las Reglas de Operación e ins-
} 
trumentos de identificación de beneficiarios de los programas; $y$ (c) crear nuevos instrumentos diseńados para aumentar las capacidades productivas de los productores pequeños con mayor grado de pobreza y vulnerabilidad, particularmente los relacionados con la comercialización (Secretaría de Desarrollo Social, 2014a).

No obstante, las Reglas de Operación 2015 del programa no permiten vislumbrar la estrategia de atención para lograr la inclusión productiva, laboral y financiera a la que hacen referencia, pues en su numeral 3.7.3.3, sobre la articulación con programas sociales relacionados con el fomento productivo, generación de ingreso e inclusión laboral, señalan:

El Programa procura la complementariedad de sus actividades con otros programas a través de modelos y esquemas de articulación con instituciones públicas, privadas y de la sociedad civil organizada que cuenten con acciones en materia de fomento productivo, generación de ingreso e inclusión laboral. En este sentido, el Programa promoverá la articulación con los Programas de Fomento Productivo de la sedesol, sagarpa, así como los que promueven la generación de ingresos, pertenecientes a la SE.

Para tales efectos, en las Reglas de Operación de los programas previstos, con la finalidad de dar un criterio de prioridad en atención a las familias Prospera, destinando los recursos para contribuir al financiamiento de las solicitudes de apoyo a sus proyectos productivos, se agregó el siguiente párrafo:

Se dará prioridad a los proyectos que cuenten con la participación de integrantes de hogares beneficiarios de Prospera [...], así como del programa piloto Territorios
Productivos, de acuerdo con la suficiencia presupuestaria. Para este criterio, el Programa determinará los recursos para financiar los proyectos demandados por dichas estrategias y, deberá, en su caso, asegurar el diseño, elaboración y acompańamiento de los proyectos para su consolidación. En los casos que sea necesario, para hacer efectivo el acceso en estos supuestos, el Programa expedirá convocatorias específicas que contendrán las acciones, recursos, metas, así como el procedimiento para el diseño, registro y operación del proyecto, ejercicio de los recursos y la comprobación de su correcta aplicación. Cuando se trate de los integrantes de hogares beneficiarios de Prospera, el Programa solicitará el número de folio familia para fines de monitoreo y seguimiento.

Adicionalmente, la Coordinación Nacional podrá establecer acciones de coordinación con otros programas de la SEP, para el intercambio de información en beneficio de las familias incorporadas a Prospera.

Por último, a pesar de la puesta en marcha de reformas estructurales relacionadas con la calidad de los servicios de educación y salud, poco se ha logrado para mejorar su calidad. El programa ha aumentado de forma significativa los tipos y montos de apoyos, así como su cobertura. Pero, sin un diagnóstico que sustente la mayoría de estos cambios, la baja cobertura y la calidad regular de los servicios de salud y, en menor medida, de educación, han limitado el impacto del PTC en mejorar el capital humano de los pobres en el desarrollo de mercados locales (Rodríguez, 2012).

Ahora bien, Tassara et al. (2015) también señalan que, a pesar de la madurez que los PTC han alcanzado en el mundo, siguen existiendo varios temas que son objeto de discusión 
entre los expertos. Entre ellos: el efectivo nivel de articulación de estos programas con los sistemas de protección social, y el diseńo y el funcionamiento de la institucionalidad de soporte. Estos dos temas de gestión han tenido un impacto significativo en el caso mexicano.

A lo largo de este artículo se han presentado algunos elementos que permiten documentar la experiencia mexicana respecto de la gestión para un sistema de protección social: la articulación del PTC con el sistema de protección social y la vinculación de los beneficiarios del PTC con las políticas públicas para acceder a mejores oportunidades de ingresos. Sin embargo, debido a la importancia que al menos las estrategias Contigo es Posible y Vivir Mejor han dado al tema territorial, en esta sección también se analizarán las mismas, aportando así a la discusión del diseño y el funcionamiento de la institucionalidad de soporte.

Respecto de la articulación del PTC con el sistema de protección social, Tassara et al. (2015) plantean que algunos analistas critican los PTC por no encarar temas de fondo, como la reforma sustantiva del mercado laboral, la redistribución equitativa del ingreso y la creación de sistemas de protección social más amplios e incluyentes.

En el caso mexicano, el sistema de protección social se ha ido conformando con programas universales o de amplia cobertura entre las personas en situación de pobreza, co- mo el Seguro Popular o la Pensión para Adultos Mayores; no obstante, estos constituyen elementos aislados, sin una visión sistémica, que al implementarse de forma articulada garanticen el acceso efectivo de la población a la protección social. Como señalan Tassara et al. (2015), "la clave parece ser la definición de objetivos claros y la articulación con sistemas de protección social integrados".

Más aún, a pesar de la existencia de elementos normativos que obligan a la creación de un padrón de beneficiarios ${ }^{6}$, en su operación se carece de una visión estratégica de integralidad de la política social. En todo caso, únicamente se establecen los mecanismos para la acumulación de información de los beneficiarios de los programas. Incluso es muy claro que la concurrencia de la política social en un sujeto de derecho se obtiene por casualidad, y no por causalidad, lo que implicaría una estrategia articulada de atención, con objetivos claros.

Por otra parte, hemos mostrado que la articulación entre la política social (representada por los beneficiarios del PTC) y la política económica se ve limitada debido a la gran cantidad de programas de desarrollo económico con escasa cobertura y mala focalización en personas en situación de pobreza y poca claridad sobre sus resultados.

Berdegué et al. (2015) señalan seis errores que tienen varias consecuencias en la política

6 La Ley General de Desarrollo Social establece, en su artículo 27, que para asegurar la equidad y eficacia de los programas de desarrollo social, la Secretaría de Desarrollo Social integrará el Padrón de los programas federales. Por ello, el 19 de diciembre de 2014 se publicaron los Lineamientos normativos para la integración del padrón único de beneficiarios. 
pública dirigida a los pequeńos productores rurales y campesinos:

1. Mala focalización: por ejemplo, al analizar el presupuesto 2007-2010 de la Secretaría de Agricultura, Ganadería, Desarrollo Rural, Pesca y Alimentación (SAGARPA), los autores muestran que los productores con predios más grandes y más capitalizados son los que reciben mayores subsidios.

2. Disociación entre desarrollo productivo y desarrollo social: las entidades con mayor número de personas en situación de pobreza reciben más apoyos con enfoque asistencial y menos recursos de la vertiente de competitividad de la Ley de Desarrollo Rural Sustentable.

3. Descoordinación entre la amplia oferta de programas: según el Catálogo de Programas y Acciones Federales y Estatales para el Desarrollo Social (CPAfEDs), existen 616 programas dirigidos al sector agropecuario, desarrollo social y desarrollo productivo $^{7}$.

4. Oferta pública federal acumulativa: según el CPAFEDs, al menos 130 programas iniciaron antes de 1985, es decir, tienen más de 30 años.

5. Ausencia de evaluaciones de impacto en programas productivos, lo que dificulta la toma de decisiones sobre cuáles programas son más efectivos.
6. Baja participación y coordinación de los gobiernos locales.

Asimismo, se mostró la importancia que en el periodo 2001-2012 tuvieron las estrategias de desarrollo social con elementos de desarrollo territorial para generar mejores ingresos, y también se evidenció el cambio de visión en el actual sexenio, dando prioridad a la seguridad alimentaria con la Cruzada $\mathrm{Na-}$ cional Contra el Hambre. En todo caso, las evaluaciones han cuestionado los resultados a causa de una insuficiente coordinación institucional y entre los tres niveles de gobierno, incluso cuando la coordinación se encarga a Comisiones existentes o creadas con el objetivo de operar la estrategia.

La coordinación de la Estrategia Microrregiones fue encargada a la Secretaría de Desarrollo Social, la cual operaba simultáneamente un programa casi homónimo (Programa de desarrollo local Microrregiones). Esta situación generó que el liderazgo de la estrategia poco a poco se fuera delegando al responsable del programa y una falta de empoderamiento para emprender de manera consistente acciones de coordinación. Es de destacarse, sin embargo, el diseño operativo y conceptual de la estrategia, el cual pone en el centro del desarrollo territorial los vínculos entre pequeńas ciudades rural-urbanas y sus relaciones con su entorno rural.

7 El Catálogo de Programas y Acciones Federales y Estatales para el Desarrollo Social es un sistema público, gubernamental y ciudadano, que clasifica la información de programas y acciones para el desarrollo social de los diferentes órdenes de gobierno y la provee en formato de datos abiertos (open data) (Sistema Nacional de Programas de Combate a la Pobreza, s.f.). 
Por otra parte, en la Estrategia 100x100 se redujo el número de municipios participantes y se encargó su operación a la Comisión Intersecretarial de Desarrollo Social, creada por mandato de la Ley General de Desarrollo Social. Sin embargo, se abandonó la estrategia de intervención definida por Microrregiones para detonar el desarrollo territorial ${ }^{8}$. Sin una estrategia de intervención y sin presupuestos establecidos para la misma, los resultados alcanzados fueron precarios.

Finalmente, la Cruzada Nacional Contra el Hambre, en su diseño y el funcionamiento de la institucionalidad de soporte, es muy parecida a la Estrategia 100x100. Para su operación se creó la Comisión Intersecretarial para la instrumentación de la Cruzada contra el Hambre, con esquemas operativos semejantes a la Comisión Intersecretarial de Desarrollo Social y, también, sin un documento público que presente la estrategia de atención.

Como señalan Tassara et al. (2015) respecto al tema de discusión sobre el diseño y funcionamiento de la institucionalidad de soporte, se han creado estructuras o comisiones con la finalidad de desempeñar una función más global en la armonización del conjunto de las políticas sociales. Sin embargo, la ausencia de una estrategia de atención bien definida, con responsables y metas, puede impedir el éxito de estos esfuerzos.

\section{CONCLUSIONES}

Durante los últimos quince años, los Gobiernos federales de México han venido planteando distintas estrategias de desarrollo social y productivo con preocupaciones y enfoques comunes, principalmente los relacionados con la formación de capacidades, la protección social, el desarrollo territorial y la generación de ingresos.

La Estrategia Contigo, implementada en el periodo 2001-2006, agrupaba las acciones del Gobierno federal en torno a las cuatro vertientes: 1) ampliación de capacidades, incluyendo educación, salud y nutrición y capacitación; 2) protección social, incluyendo previsión social y protección contra riesgos individuales y colectivos; 3) generación de oportunidades de ingreso, incluyendo desarrollo local y acceso al crédito, así como generación de empleo; y 4) formación de patrimonio, incluyendo ahorro, vivienda y derechos de propiedad.

La Estrategia Vivir Mejor, implementada en el periodo 2006-2012, también agrupaba líneas de acción similares para el desarrollo humano sustentable: 1) impulsar el desarrollo de las capacidades de los mexicanos; 2) proporcionar una red de protección social; y3) facilitar el acceso al empleo formal a todas las mexicanas y los mexicanos, fortaleciendo

\footnotetext{
8 Según la estrategia de atención, dotar a las localidades estratégicas de servicios de electricidad, salud, telefonía, caminos, saneamiento, educación, agua potable, abasto de productos básicos y piso firme, así como de Centros Comunitarios de Aprendizaje y, principalmente, la existencia de circuitos de actividad productiva, serían detonantes del desarrollo territorial.
} 
la coordinación entre la política social y la política económica.

Por su parte, en la Cruzada Nacional Contra el Hambre, los objetivos se modifican para poner en el centro la alimentación, la nutrición, la producción de alimentos, la participación ciudadana y los ingresos de los pequeños productores, sin hacer una mención específica a la protección social o al desarrollo territorial.

Un elemento común a estas tres estrategias es la mención constante a la coordinación institucional y con los niveles de Gobierno como parte de las acciones necesarias para su éxito. También se destaca la importancia otorgada al PTC de turno, a la protección social y -al menos en las dos primeras estrategiasal desarrollo territorial y a la generación de ingresos.

Progresa, desde su creación en 1997, ha tenido una amplia aceptación por sus resultados en la generación de capacidades básicas en materia de educación, salud y alimentación. No obstante, los efectos de los cambios posteriores en su diseño no han sido necesariamente estudiados con el mismo rigor, por ejemplo, aquellos relacionados con la expansión del programa a zonas urbanas o la creación de nuevos componentes en el marco de Oportunidades o del actual Prospera. A pesar de esta falta, los PTC siguen siendo el elemento central de la política social, con una cobertura actual de 6,1 millones de familias.

Cada cambio de Gobierno se ha caracterizado también por la incorporación de nuevos programas como el Seguro Popular, las pensiones para adultos mayores, programas de estancias infantiles y seguros de vida para jefas de familia. Las evaluaciones de estos programas destacan impactos en sus principales variables de interés. Por otra parte, no existe una estrategia clara de vinculación entre los beneficiarios del PTC y de los programas de protección social.

Además del PTC y de los programas de protección social, en el periodo 2001-2012, los planes de Gobierno incluyeron estrategias de desarrollo social con elementos de desarrollo territorial para generar mejores ingresos. El presidente Vicente Fox implementó la Estrategia Microrregiones, cuyo objetivo era superar la pobreza y promover el desarrollo integral en municipios de alta y muy alta marginación. Posteriormente, la Estrategia 100x100 del presidente Calderón, se enfocó a las condiciones de rezago de los municipios con menor índice de desarrollo humano (IDH) del país. En ambos casos, además del PTC, estas estrategias fueron la principal herramienta para reducir disparidades desde la política social, pero en el sexenio del presidente Enrique Peńa Nieto, esta visión cambió y se dio prioridad a la seguridad alimentaria estableciendo la Cruzada Nacional Contra el Hambre. Como se ha mencionado, las deficiencias en la coordinación institucional e intergubernamental han sido señaladas en las evaluaciones correspondientes. Esta situación prevalece incluso cuando se responsabiliza de la coordinación a Comisiones con encomiendas públicas para operar las estrategias.

Por otra parte, la articulación entre la política social (representada por los beneficiarios del PTC) y la política económica se ve limitada debido a la gran cantidad de programas de desarrollo económico con escasa cobertura 
y mala focalización a personas en situación de pobreza. La insuficiencia de evaluaciones y conocimiento sobre alternativas de atención ha llevado a la creación de un programa piloto que busca dar respuesta al desarrollo territorial con vínculos entre las ciudades intermedias y las localidades rurales, así como al acceso efectivo a la política de desarrollo económico mediante la articulación de la demanda social. Este programa deberá mostrar resultados para poder ser considerado una estrategia de atención susceptible de expansión y réplica.

\section{REFERENCIAS}

Adato, M. (2000). The Impact of Progresa on Community Social Relationships. Washington DC: International Food Policy Research Institute (IFPRI).

Adato, M., de la Brière, K. B., Mindek, D. y Quisumbing, A. (2000). Final Report: The Impact of Progresa on Women's Status and Intrahousehold Relations. Washington DC: IFPRI.

Banegas, I. (2013). Evaluación especifica de desempeño del Programa 70 y más/Pensión para Adultos Mayores 2012-2013. México DF: Universidad Nacional Autónoma de México.

Banegas, I. (2015). Evaluación especifica de desempeño del Programa Seguro de Vida a Jefas de Familia 2014-2015. México DF: Universidad Nacional Autónoma de México.

Behrman, J. R. y Todd, P. E. (1999). Randomness in the Experimental Samples of Progresa (Education, Health and Nutrition Program). Washington DC: IFPRI.

Behrman, J. y Hoddinott, J. (2000). An Evaluation of the Impact of Progresa on Pre-school Child Height. Washington DC: IFPRI.
Behrman, J., Sengupta, P. y Todd, P. (2000). The Impact of Progresa on Achievement Test Scores in the First Year. Washington DC: IFPRI.

Behrman, J., Sengupta, P. y Todd, P. (2001). Progressing through Progresa: An Impact Assessment of a School Subsidy Experiment. Washington DC: Universidad de Pennsylvania \& IFPRI.

Berdegué, J., Franco, G., Gordillo, G., Robles, H., Scott, J., Soloaga, I., Toledo, C. y Yúnez-Naude, A. (2015). Territorios productivos. Un programa articulador para reducir la pobreza rural a través el incremento de la productividad, la producción y los ingresos. Centro Latinoamericano para el Desarrollo Rural (RIMISP), Documento de trabajo 131. Grupo de Trabajo Desarrollo con Cohesión Territorial.

Chacón, B. (2014). Estudio comparativo de la politica de desarrollo social en México. México DF: Fundación Rafael Preciado Hernández, Documento de trabajo 520.

Conde, C. (2006). Evaluación de consistencia y resultados del Programa Vivienda Rural 2005. México DF: El Colegio Mexiquense.

Comisión Nacional de Protección Social en Salud (2005). Reporte No. 6. Recuperado de http:// www.salud.gob.mx/transparencia/informes/ segpopular/Informe_segundo_semestre2005.pdf CONEVAL (2008). Informe de evaluación de la política de desarrollo social en México 2008. México DF: CONEVAL.

coneval (2013a). Diagnóstico del diseño de la Cruzada Nacional contra el Hambre. México DF: CONEVAL.

CONEVAL (2013b). Esquema general de la evaluación de la Cruzada Nacional contra el Hambre 2013-2019. México DF: CONEVAL.

CONEVAL (2013c). Evaluación de impacto de la Estrategia 100x100. México DF: CONEVAL. 
CONEVAl (2015). Informe de resultados intermedios de la Cruzada Nacional contra el Hambre. México DF: CONEVAL.

Curristine, T. (2014). Institutionalizing Performance Budgeting: Key Institutions and Actors-Roles and Incentive Structures. Washington DC: International Monetary Fund, Fiscal Affairs Department. FMs (2004). Evaluación externa del Programa de Ahorro, Subsidio y Crédito para la Vivienda Progresiva Tu Casa 2003. México DF: Fundación Mexicana para la Salud (FMs).

Gertler, P. J. (2000). Final Report: The Impact of Progresa on Health. Washington DC: IFPRI.

Grupo de Diálogo Rural (coord.) (2012). Conocimiento y cambio en pobreza rural y desarrollo. Elementos para la construcción de una politica. México DF: UNAM.

Handa, S., Huerta, M. C., Pérez, R. y Straffon, B. (2000). Final Report: Poverty, Inequality, and "Spill-Over" in Mexico's Education, Health, and Nutrition Program. Washington DC: IFPRI.

Hoddinott, J., Skoufias, E. y Washburn, R. (2000). The Impact of Progresa on Consumption: A Final Report. September. Washington DC: IFPRI.

Hatry, H. (2014). Transforming Performance Measurement for the 21st Century. Washington DC: The Urban Institute.

Levy, S. y Rodríguez, E. (2005). Sin herencia de pobreza: el programa Progresa - Oportunidades de México. Washington DC: Banco Interamericano de Desarrollo (BID).

Levy, S. (2007). Productividad, crecimiento y pobreza en México: ¿qué sigue después de Progresa-Oportunidades? Washington DC: BID.

Marcel, M. (2009). Presupuesto por resultados: ¿Moda burocrática o nuevo paradigma en gestión pública? Washington DC: BID.
Mujica, R. (2007). Estrategia Microrregiones. México DF: Secretaría de Desarrollo Social.

Parker, S. y Skoufias, E. (2000). Final Report: The Impact of Progresa on Work, Leisure, and Time Allocation. Washington DC: IFPRI.

Parker, S. y Scott, J. (2001). Evaluación del Programa de Educación, Saludy Alimentación (Progresa) a partir de: Indicadores de Seguimiento, Evaluación y Gestión 1998-2001 y Encuestas de Evaluación 2000. México DF: Centro de Investigación y Docencia Económicas (CIDE).

Rawlings, L. y Rubio, G. (2003). Evaluación del impacto de los programas de transferencias condicionadas en efectivo. México DF: Secretaría de Desarrollo Social, Serie Cuadernos de Desarrollo Humano 10.

Rodríguez, E. (2013). Evaluación especifica de desempeño del Programa de Desarrollo Humano Oportunidades 2012-2013. México DF: Hospital Infantil Federico Gómez.

Schultz, T. P. (2000a). Impact of Progresa on School Attendance Rates in the Sampled Population. Washington DC: IFPRI.

Schultz, T. P. (2000b). School Subsidies for the Poor: Evaluating a Mexican Strategy for Reducing Poverty. Washington DC: IFPRI.

Schultz, T. P. (2000c). Final Report: The Impact of Progresa on School Enrollments. Washington DC: IFPRI.

Schultz, T. P. (2001). School Subsidies for the Poor: Evaluating the Mexican Progresa Poverty Program. New Haven (Ст): Yale University Economic Growth Center.

Székely, M. (2003). Estrategia Contigo en Memorias de la Conferencia Internacional México 2003, mejores prácticas de politica Social. México DF: Banco Mundial y Secretaría de Desarrollo Social.

Skoufias, E. (2001). Progresa and its Impacts on the Human Capital and Welfare of Households in Rural 
Mexico: A Synthesis of the Results of an Evaluation by IFPRI. Washington DC: IFPRI.

Skoufias, E., Davis, B. y Behrman, J. (1999). Final Report: An Evaluation of the Selection of Benefciary Households in the Education, Health, and Nutrition Program (Progresa) of Mexico. Washington DC: IFPRI.

Skoufias, E., Davis, B. y de la Vega, S. (1999). An Addendum to the Final Report: An Evaluation of the Selection of Beneficiary Households in the Education, Health, and Nutrition Program (Progresa) of Mexico. Targeting the Poor in Mexico: Evaluation of the Selection of Beneficiary Households into Progresa. Washington DC: IFPRI.

Skoufias, E. y McClafferty, B. (2001). Is Progresa Working? Summary of the Results of an Evaluation. Washington DC: IFPRI.

Secretaría de Desarrollo Social (2002). Acuerdo por el que se emiten las Reglas de Operación de los Programas de Subsidios del Ramo Administrativo 20 Desarrollo Social, del Presupuesto de Egresos de la Federación para el Ejercicio Fiscal 2002. Diario Oficial de la Federación.

Secretaría de Desarrollo Social (2014a). Decreto por el que se crea la Coordinación Nacional de Prospera Programa de Inclusión Social. Diario Oficial de la Federación.

Secretaría de Desarrollo Social (2014b). Acuerdo por el que se emiten las Reglas de Operación de Prospera Programa de Inclusión Social, para el ejercicio fiscal 2015. Diario Oficial de la Federación.

Secretaría de Desarrollo Social (2014c). Diagnóstico y propuesta de atención del Programa de Comedores Comunitarios. México DF: Secretaría de Desarrollo Social.
Secretaría de Desarrollo Social (2015a). Lineamientos especificos del Programa de Comedores Comunitarios, en el marco de la Cruzada contra el Hambre. México DF: Secretaría de Desarrollo Social.

Secretaría de Desarrollo Social (2015b). Logros 2014 del Programa Nacional México sin Hambre. México DF: Secretaría de Desarrollo Social.

Secretaría de Hacienda y Crédito Público (2016). Distribución del pago de impuestos y recepción del gasto público por deciles de hogares y personas para el año 2014. México DF: Secretaría de Hacienda y Crédito Público.

Sistema Nacional de Programas de combate a la pobreza (s.f.). Index. Recuperado de http://www. programassociales.mx/

Soto, J. (2012). Meta Evaluación 2007-2012 del Programa 70 y Más. Tuxla Gutiérrez: Universidad Autónoma de Chiapas, Centro Universidad-Empresa.

Tassara, C., Ibarra, A. y Vargas, L. H. (2015). Protección socialy lucha contra la pobreza en Brasil, Colombia y Chile. ¿Graduarse de los PTC o salir de la pobreza? Madrid: Programa eurosocial.

Téllez, M. (2010). Evaluación complementaria de impacto del Programa 70 y Más. México DF: Instituto Nacional de Salud Pública.

Teruel, G. y Davis, B. (2000). Final Report: An Evaluation of the Impact of Progresa Cash Payments on Private Inter-Household Transfers. Washington DC: IFPRI.

Urbina, M. (2015). Evaluación especifica de desempeño del Programa Seguro Popular 2014-2015. México DF: Investigación en Salud y Demografía (INSAD). 